\title{
Type II SN Light Curves from the Caltech Core Collapse Project
}

\section{Iair Arcavi}

Department of Particle Physics and Astrophysics, The Weizmann Institute of Science, Rehovot 76100, Israel

email: iair.arcavi@weizmann.ac.il

We presented our analysis of a sample of type II supernova (SN) light curves measured by the Caltech Core Collapse Project (CCCP). CCCP is a large observational program which made use of the robotic 60 -in and the Hale 200 -in telescopes to obtain optical photometry, spectroscopy and IR photometry of 49 nearby core-collapse supernovae (SNe). It provides a fair sample of core-collapse events, with well-defined selection criteria, and uniform, high-quality optical/IR observations. Our goal is to characterize the little-studied properties of core-collapse supernovae as a population. Preliminary data indicate a diverse set of sub-populations including "standard" type IIP supernovæ, declining supernovæ (at different rates) and slowly rising peculiar supernovæ. Work is in progress to map and quantify that diversity better. It is hoped that a single tunable formula will be able to describe most light-curve shapes, thereby helping us attain a better understanding of the physical mechanisms underlying these results.

\section{Stopping and Looking: On Error Bars in Time Series}

Guillaume Belanger

ESAC, Villanueva de la Cañada, 28691 Madrid, Spain

email: guillaume.belanger@esa.int

In X-ray Astronomy, the error bar or uncertainty associated with the value of a bin in a time series is usually calculated from the square root of the number of events in that bin. This standard practice is erroneous both on theoretical and on practical grounds. On theoretical grounds, it fails to conform to the fact that the uncertainty on a measurement depends upon the accuracy with which the measurement is made, not on the value measured. On practical grounds, it fails to give the correct value of the mean count rate when the error bars are taken into account. Therefore, this practice should be abandoned in favour of an alternative where the error bar is a more appropriate representation of the uncertainty on the measurement, and therefore it does not depend upon the number of events in the given bin. 


\title{
Photometric Reverberation Mapping of the Broad Emission Line Region in AGN
}

\author{
Doron Chelouche \\ Department of Physics, University of Haifa, Mount Carmel, Haifa 31905, Israel \\ email: doron@ias.edu
}

Measuring black hole (BH) masses in galaxies has considerable implications for our understanding of galaxy formation and evolution and accretion physics in active galactic nuclei (AGN). A reliable method for estimating the black-hole mass in AGN uses reverberation mapping, which allows one to measure the size of the broad emission line region in those objects. Presently, the mass of $<50$ low- $z$ BHs has been measured in this way. The poster proposed a new method for measuring the size of the broad emission-line region (BLR) using broad-band photometric light curves. Applying the method to a subsample of AGN with previously measured BLR sizes gives consistent results. The poster reported new $\mathrm{BH}$ mass measurements that use currently available photometric data, and single-epoch spectral information. This opens up the possibility of measuring BLR sizes and $\mathrm{BH}$ masses in numerous objects, and over a broad redshift range, using upcoming photometric surveys.

\section{Identification and Investigation of "Primitive Emitters" that Form Prompt Phases of GRBs}

\author{
Anton Chernenko \\ Space Research Institute, Russian Academy of Sciences, 84/32, Moscow 117997, Russia \\ email: anton@cgrsmx.iki.rssi.ru
}

GRBs are one of the most diverse celestial objects in terms of their time histories and spectral variability during prompt phases. A number of peaks with durations ranging from milliseconds to tens of seconds within an individual GRB randomly overlap, and possibilities to investigate spectral evolution of single unambiguously isolated emission episodes are scarce. Today, no solid theoretical framework for explaining prompt emission of GRBs exists, and no single emission mechanism can explain the spectral evolution of the entire prompt phase of a given GRB. An attempt to fit gamma-rays by a theoretical spectral shape based on particular emission mechanisms imposes rather weak constraints on the model parameters since, owing to the overlapping nature of emission, that fit needs to involve several components. Moreover, the statistics of available gamma-ray data hardly allow one to distinguish between models with the number of variable parameters greater than two. Therefore, a possibility to identify and study emission episodes that are unambiguously produced by single physical emitters is very important from the theoretical perspective. We found that in almost any GRB there is a number of time intervals of considerable duration (up to tens of seconds) during which emission is highly variable in terms of flux, but manifests very little spectral variability - its spectral shape stays constant. On the other hand, spectra of different intervals differ significantly from each other. Such intervals often correspond to the few initial seconds of GRBs and to their tails, but frequently they also span periods with complex multi-peak structures in the middle of the prompt phase. Spectrum-flux correlations are natural in either synchrotron or IC models, and the very existence of periods where such correlations are minimal is important since it implies existence of special "extensive" emission régimes within those models. It is also unlikely that, during such a period, any overlapping takes place. Two or more emitters, even with constant spectra, should be connected too tightly to produce 
such a coherent picture. It is therefore likely that we are rather frequently able to observe what we call individual "primitive emitters" whose spectra and time histories could be studied independently from other emitters. The poster present the results of this study.

\section{ULTRACAM Observations of Ultra-Compact Binaries}

C. Copperwheat ${ }^{1}$, T. Marsh ${ }^{1}$, V. Dhillon ${ }^{2}$, S. Littlefair ${ }^{2}$, and D. Steeghs ${ }^{1}$

${ }^{1}$ Department of Physics, University of Warwick, Coventry CV4 7AL, UK

email: c. copperwheat@warwick.ac.uk

${ }^{2}$ Department of Physics and Astronomy, University of Sheffield, Sheffield S3 7RH, UK

The AM Canum Venaticorum (AM CVn) stars are ultra-compact binaries with the lowest periods of any binary subclass, and consist of a white dwarf accreting material from a donor star that is itself fully or partially degenerate. These objects offer new insight into the formation and evolution of binary systems, and are predicted to be among the strongest gravitational-wave sources in the sky. The poster presented multi-band, high time-resolution light curves of a number of these systems, obtained with the fast photometer ULTRACAM. It discussed the 28-min binary SDSS 0926+3624, the only known eclipsing source of this class. From light-curve models we make the most precise parameter determinations for any $\mathrm{AM} \mathrm{CVn}$, and determine the degree of degeneracy of the donor star - a key parameter in differentiating between the proposed formation paths for these objects. The poster also discussed additional phenomena that are apparent in the optical data for this system, such as the superhump, quasi-periodic oscillations, and the outbursting behaviour. In conclusion it outlined our long-term timing programme, through which we are measuring the period evolution of a number of different AM CVn systems. The two most compact systems (HM Cnc and V407 Vul) are known to be decreasing in period, which is the opposite of what was initially expected for accreting AM CVns. For those systems we aim to measure eventually the second time-derivative of the orbital period, which will differentiate between the various formation models. For the 10-min binary ES Cet the poster showed how ten years of timing measurements suggest a double white-dwarf formation path for that system.

\section{Multi-Wavelength Stellar Variability in the Time-Domain Era}

James Davenport ${ }^{1}$ et al.

${ }^{1}$ Department of Astronomy, University of Washington, Seattle, WA 98195, USA email: jrad@astro.washington.edu

We presented results from our studies of (primarily low-mass) main-sequence stars using the SDSS Stripe 82, 2MASS Calibration Fields, and WISE time-domain databases. These photometric catalogues provide measurements from the last decade ranging in wavelength from 0.36 to $22 \mu$. Many forms of variability for these stars are best identified in certain wavelength régimes, and the levels of variability differ across the many photometry surveys. We have studied the data to characterize the rates of flares, periodic sources such as eclipsing binaries, and also the variability structure in the quiescent nonflaring state. Comparisons between wide-field survey data and dedicated ground- and space-based photometric monitoring were also presented. 


\section{Contact Binary Population from the RATS Survey}

P. Hakala ${ }^{1}$, G. Ramsay ${ }^{2}$, and T. Barclay ${ }^{2,3}$

${ }^{1}$ FINCA, University of Turku, F-21500 PIIKKIÖ, Finland

email: pahakala@astro.helsinki.fi

${ }^{2}$ Armagh Observatory, College Hill, Armagh BT61 9DG, UK

${ }^{3}$ MSSL, University College London, Holmbury St. Mary, Dorking, RH5 6NT, UK

We presented the first results from our modelling of the Contact Binary population of our variability survey by the $40 \mathrm{deg}^{2}$ RATS. We matched the partial light curves against a vast library of pre-computed light-curve models, and derived approximate system parameters for hundreds of sources.

\section{Visibility Stacking in the Quest for Radio Emission from SNIa}

Paul Hancock, Bryan Gaensler, and Tara Murphy

Sydney Institute for Astronomy, The University of Sydney, NSW 2006, Australia

email: jancock@physics.usyd.edu.au

The poster described the process of stacking radio interferometry visibilities to form a deep composite image, and its application to the observation of transient phenomena. We applied "visibility stacking" to 46 archival Very Large Array observations of nearby type Ia supernovæ (SNeIa). This new approach provides an upper limit on the SNIa ensemble peak radio luminosity of $1.2 \times 10^{25} \mathrm{erg} \mathrm{s}^{-1} \mathrm{~Hz}^{-1}$ at $5 \mathrm{GHz}$, which is $5-10$ times lower than previously measured. That luminosity implies an upper limit on the average companion stellar wind mass-loss rate of $1.3 \times 10^{-7} M_{\odot} \mathrm{yr}^{-1}$. This mass loss rate is consistent with the double degenerate model for SNeIa, and rules out intermediate and high-mass companions in the single degenerate one. In the era of time-domain astronomy, techniques such as visibility stacking will be important in extracting the maximum amount of information from observations of populations of short-lived events.

\section{Stellar Flares in Time Domain Surveys}

Eric J. Hilton ${ }^{1}$, Suzanne L. Hawley ${ }^{2}$, and Adam F. Kowalski ${ }^{2}$

1 University of Hawaii at Manao, Honolulu, HI 96822, USA

${ }^{2}$ Astronomy Department, University of Washington, Seattle, WA 98915, USA email: hilton@ifa.hawaii.edu

Stellar flares are powerful magnetic reconnection events that cause transient flux enhancements of up to several magnitudes on low-mass stars. The flares have a characteristic blue colour, and the largest ones may last many hours. Small flares may occur several times per hour. The poster presented simulations of the observational signatures of flares in time-domain surveys using new measurements of flare rates on low-mass stars in a variety of filters. We paid particular attention to the rate of optical transients, where a flare occurs on a previously undetected source. In the poster we compared our model to previous survey data, and predicted flare rates in upcoming surveys. New large surveys will allow us to determine how the flare rates, and hence magnetic fields, change as a function of stellar temperature, age and other physical parameters. 


\section{Low-Frequency Searches for Radio Transient Emission using the VLA}

T. Jaeger ${ }^{1}$, N. Kassim ${ }^{1}$, S. Hyman ${ }^{2}$, J. Lazio ${ }^{3}$, and R. Osten ${ }^{4}$

${ }^{1}$ US Naval Research Laboratory, Washington, DC 20375, USA

email: ted.jaeger.ctr@nrl.navy.mil

${ }^{2}$ Dept. of Physics and Engineering, Sweet Briar College, Sweet Briar, VA 24595, USA

${ }^{3}$ Jet Propulsion Laboratory, Pasadena, CA 91109, USA

${ }^{4}$ Space Telescope Science Institute, Baltimore, MD 21218, USA

A variety of sources have been predicted to emit at metre wavelengths and would probably appear as transients, including bursts from extrasolar planets and brown dwarfs and prompt emission from gamma-ray bursts. Low-frequency VLA observations are well suited to probe the dynamic radio sky, given the the large field of view $\left(>150 \mathrm{deg}^{2}\right)$ at $74 \mathrm{MHz}$ and sub-mJy sensitivity at $325 \mathrm{MHz}$. The poster presented preliminary results from multiple low-frequency radio transient searches using data from the VLA archive. In particular, it reported on our searches at $325 \mathrm{MHz}$ using targeted fields, and an allsky search using fields from the 74-MHz VLA Low-frequency Sky Survey (VLSS), and discussed implications of any transient non-detection for the rate of gamma-ray bursts and/or radio supernovae.

\section{The Century Before Photography}

Derek Jones

Institute of Astronomy, Madingley Road, Cambridge, CB3 OHA, UK

email: dhpj@ast.cam.ac.uk

The concept of "flux-limited sample" was developed by radio astronomers after World War II, but the same idea can be traced through the work of the optical astronomers: the Herschels (father and son), Lalande, Bessel and Argelander. One motivation was the search for minor planets; variable stars were then regarded as a nuisance. The perennial difficulty was the lack of a consistent definition of stellar magnitude.

\section{Automatic Real-Time Modelling of Anomalous Microlensing Events}

N. Kains ${ }^{1}$, P. Browne ${ }^{1}$, K. Horne ${ }^{1}$, and A. Cassan ${ }^{2}$

${ }^{1}$ School of Physics and Astronomy, University of St Andrews, Fife, KY16 9SS, UK email: nk87@st-and.ac.uk

${ }^{2}$ Institut d'Astrophysique de Paris, 98 bis Boulevard Arago, 75014 Paris, France

Gravitational microlensing is a well-established planet-detection method, with notable discoveries including the first cool, rocky exoplanet. Today, new-generation surveys and robotic telescopes allow us to monitor and follow up thousands of microlensing events per year, of which a significant fraction is "anomalous". Each anomalous microlensing event traditionally requires significant computational and human resources to model, and this is difficult to achieve on the short timescales of the typical microlensing anomalies, particularly those of possible planetary origin. The poster presented the development of automatised methods to model anomalous microlensing events so as to provide feedback to both robotic and human observers about the nature of on-going events. 


\section{Wide and Fast: Monitoring the Sky in the Sub-Second Domain}

S. Karpov ${ }^{1}$ et al.

${ }^{1}$ Special Astrophysical Observatory of RAS, Karachaevo-Cherkesia 369167, Russia email: karpov@sao.ru

The poster presented our long-term efforts to perform optical wide-field monitoring of the sky with sub-second temporal resolution, in order to look for optical components of GRBs, fast-moving satellites, and meteors. It described the concepts and the results of operating our wide-field cameras, FAVOR and TORTORA, and included observations of the complete development of optical emission from the Naked-Eye Burst, which has provided significant insight into the nature of its central engine. The poster also gave a status report on our new-generation instrument, MiniMegaTORTORA, a multi-objective transforming camera which is able both to detect and to classify fast optical transients and also to perform colour and polarimetric observations of them. The instrument is currently being commissioned.

\section{The Caltech Blazar Monitoring Programme}

O. G. King ${ }^{1}$ (for the OVRO 40-m Collaboration)

${ }^{1}$ Keck Institute for Space Studies, California Institute of Technology

Pasadena, CA 91125, USA

email: ogk@astro.caltech.edu

The OVRO 40-m Telescope monitoring program currently carries out twice-weekly measurements of the 15-GHz flux density of nearly 1600 blazars and other AGN, including all those associated with northern detections (declination $>-20^{\circ}$ ) by the FERMI Large Area Telescope (LAT) and a pre-selected sample ideal for statistical studies. The poster presented some results from the programme, and described the statistical methods we have developed to study the intrinsic variability of blazar populations.

\section{Anomalously Intense Pulses from Pulsars with LOFAR LBAs}

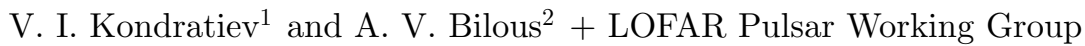

${ }^{1}$ Netherlands Institute for Radio Astronomy, 7990 AA Dwingeloo, The Netherlands

${ }^{2}$ Department of Astronomy, University of Virginia, Charlottesville, VA 22904, USA email: kondratiev@astron.nl

The poster reported the results of our observations of anomalously intensive pulses from several pulsars with the LOFAR, in the frequency range 20-70 MHz. Such pulses were first discovered by Ul'yanov et al. in 2006 using the UTR-2 radio telescope at frequencies below $30 \mathrm{MHz}$; the intensity of that source was shown to be strongly modulated in both time and frequency. LOFAR's much broader frequency range allows one to study such pulses in more detail. The poster discussed the possible origin of the pulses and their relation to the giant-pulse phenomenon observed in other pulsars at higher frequencies. 


\section{The "SED" Machine}

Nick Konidaris $^{1}$, Sagi Ben-Ami ${ }^{2}$, Robert Quimby ${ }^{1}$, and Shri Kulkarni ${ }^{1}$

${ }^{1}$ California Institute of Technology, Pasadena, CA 91125, USA

2 The Weizmann Institute of Science, Rehovot 76100, Israel

email: npk@astro.caltech.exu

The poster described the "SED Machine", a spectrograph designed around the goal of classifying transients efficiently.

\section{Gamma-Ray Variability of Blazars Observed with FERMI LAT}

Stefan Larsson ${ }^{1}$, Stefano Ciprini, Lise Escande, Benoit Lott, and Greg Madejski (on behalf of the FERMi LAT collaboration)

${ }^{1}$ Department of Astronomy, Stockholm University, SE-106 91 Stockholm, Sweden

email: stefan@astro.su.se

Since its launch in 2008 the FERMI Large Area Telescope (LAT) has provided regular monitoring of the gamma-ray emission from blazars on time-scales from hours to years. By combining observations at other wavelengths, it is now possible to study variability and correlation properties in a much more systematic and detailed way than ever before. The poster described FERMI LAT observations of blazars, the properties and limitations of produced light curves, and how those can be handled. Results from timing analyses were also presented; they could be related to variability behaviour at other wavelengths, and to models of those sources.

\section{Ultra-Compact White Dwarf Binaries from the Palomar Transient Factory}

David Levitan ${ }^{1}$ et al. (on behalf of the Palomar Transient Factory Collaboration)

${ }^{1}$ California Institute of Technology, Pasadena, CA 91125, USA

email: dlevitan@caltech.edu

The rise of large-area synoptic surveys has enabled the efficient discovery of ultra-compact white-dwarf binaries. Both outbursting systems (including Cataclysmic Variables and AM CVn systems) and eclipsing systems (such as detached white-dwarf binaries) are targets of new surveys such as the Palomar Transient Factory (PTF). The poster described our search for those systems with the PTF. It outlined the motivation for our search and summarized our discoveries to date; the latter included numerous cataclysmic variables and four of the very rare AM CVn systems. It commented on the techniques we are using to conduct our search, and the follow-up efforts involved. It concluded by discussimg the future potential of such surveys, and provided some estimates of the number of ultra-compact systems that the PTF may discover. 


\title{
Modelling the Light Curve of Tidal Disruption Events
}

Giuseppe Lodato

Universit degli Studi di Milano, Dipartimento di Fisica, I-20133 Milano, Italy

email: giuseppe.lodato@unimi.it

The tidal disruption of stars in the gravitational field of a supermassive black hole is a useful tool to determine the properties of central black holes in quiescent galaxies; it can also probe stellar dynamics in galactic nuclei. For many years the theoretical understanding of such events was largely based on the pioneering models of Rees and Phinney, who predicted that the light curve should scale as $\sim t^{(-5 / 3)}$. The poster revisited the issue, taking into account two new results: (a) recent numerical simulations have indicated that, especially at early times, the evolution of the infall rate of the debris can show significant departures from the standard profile, depending on the type of star being disrupted, and (b) the monochromatic light curves at specific wavelengths can be quite different from the bolometric one, which should scale with the infall rate but can be affected by the presence of a significant wind emission for strongly super-Eddington events. These issues were also discussed in the light of recently observed candidate TDEs.

\section{ASTROSAT: The Indian Multi-Wavelength Astronomy Satellite}

R. K. Manchanda ${ }^{1}$ (on behalf of Astrosat payload teams)

${ }^{1}$ Tata Institute of Fundamental Research, Colaba, Mumbai 400 005, India email: ravi@tifr.res.in

The Indian Astronomy satellite, code named ASTROSAT, is designed to carry out multiwavelength studies of a variety of Galactic and extragalactic sources from the UV band (1000-3000 $\AA$ ), the optical band, and the soft and hard X-ray bands covering the range 0.3-80 keV. The planned X-ray studies will span imaging plus spectral and temporal variations of Galactic and extragalactic sources. Five different payloads presently under development will achieve those mission targets. The satellite will be launched in mid-2012.

\section{The Near-Infrared Variability of Quasars}

\author{
A. A. Miller, J. S. Bloom, and N. R. Butler \\ Astronomy Department, University of California, Berkeley, CA 94720, USA ) \\ email: amiller@astro.berkeley.edu
}

The poster explored the intrinsic variability of quasi-stellar objects (QSOs) that had been observed as part of the NEWFIRM Medium Band Survey (MBS). We had used data obtained in 6 filters between 1-2.2 $\mu$, and parameterized the structure function for variability in each of the filters for the 40 or so known QSOs that were observed. The poster described how we had compared the variability to that of known variable stars and had examined the possibility of selecting QSOs from near-infrared (NIR) variability alone, regardless of colour. Within the context of upcoming NIR synoptic surveys such as WFIRST and SASIR, we had projected the efficiency with which QSOs can be discovered on the basis of their intrinsic variability. 


\section{Fallback Supernovæ: Fast \& Faint Domain of Core-Collapse Supernovæ}

Takashi Moriya

Institute for the Physics and Mathematics of the Universe, University of Tokyo, Kashiwa, Chiba 277-8583, Japan

email: takashi.moriya@ipmu.jp

Supernovæ (SNe) from massive stars (core-collapse $\mathrm{SNe}$ ) are rich in variety. Many kinds of light curves and spectral types emerge from massive stars depending primarily on their main-sequence mass. Very massive stars are expected not to end up as SNe because all the materials are collapsed into the central black hole. However, if the central black hole is not massive enough, some part of the progenitors can escape from the black hole and can potentially be observed as SNe (fallback SNe). We have performed numerical simulations of fallback SNe, and found that many fallback SNe are predicted to be observable during the rapid brightness rise (which is sometimes less than 10 days) as faint SNe. The poster compared our model with the faint and fast evolved supernova SN 2008ha, and showed that SN 2008ha is consistent with our model of fallback SNe.

\section{Subaru Wide-Field Variability Survey for Active Galactic Nuclei}

Tomoki Morokuma ${ }^{1}$ et al.

${ }^{1}$ Institute of Astronomy, University of Tokyo, Mitaka, Tokyo 181-0015, Japan email: tmorokuma@ioa.s.u-tokyo.ac.jp

The poster presented our survey for active galactic nuclei (AGN) found via optical variability with a wide-field optical imager, "Suprime-Cam", on the prime focus of the 8.2-m Subaru telescope. By combining our observations with 50-100-ksec X-ray exposures obtained with XMM-Newton, we confirmed that optical variability is a useful tool for finding AGN. We had also found that it plays a complementary and important role in the study of low-luminosity AGN for which there are X-ray observations. A significant fraction of AGN selected by their optical variability are below the X-ray detection limit; that had also been shown by studies with HST. The poster showed the result of applying our method to the same parent sample at the faint end of the quasar luminosity function at high red-shifts. It concluded with a brief introduction of the next-generation wide-field imager, "Hyper Suprime-Cam" (HSC, which was installed on the Subaru telescope in 2011), a description of our survey plan, and some results expected on the topic of AGN variability. 


\section{Real-Time Analysis in the Palomar Transient Factory}

Peter Nugent ${ }^{1,2}$

${ }^{1}$ University of California, Berkeley, Berkeley CA, 94720, USA

${ }^{2}$ Physics Division, Lawrence Berkeley National Laboratory, Berkeley, CA 94720, USA email: penugent@lbl.gov

The Palomar Transient Factory (PTF) is an experiment designed to explore systematically the optical transient and variable sky. The main goal of this project is to fill the gaps in our present-day knowledge of the optical transient phase space. Besides reasonably well-studied populations (such as classical novæ and supernovæ), there exist many types of either poorly-constrained events (such as luminous red novae and tidal disruption flares) or predicted but not yet discovered phenomena (such as orphan afterglows of gamma-ray bursts). Beginning in May of 2010, we put into action an end-to-end near real-time pipeline for the discovery and automated follow-up of new transient sources. The poster discussed the challenges we faced in enacting such a pipeline, described our successes over the first year since implementation, and outlined directions for future improvements.

\section{The Long-Term Optical Polarization Variability of the BL Lac Object PKS 2155-304}

N. Pekeur ${ }^{1}$, S. B. Potter ${ }^{2}$, P. M. Chadwick ${ }^{1}$, and M. Daniel ${ }^{1}$

${ }^{1}$ Department of Physics, University of Durham, Durham DH1 3LE, UK

email: n.w.pekeur@durham.ac.uk

${ }^{2}$ South African Astronomical Observatory, Cape Town, South Africa

Polarization provides a direct means of disentangling the thermal emission of blazars from the synchrotron emission at optical wavelengths. The poster presented the optical polarization light curve of the high-frequency peaked blazar PKS 2155-304 during 2009 and 2010, when the source experienced both active and quiescent periods. Measurements were obtained with the HIgh Speed Photo-POlarimeter (HIPPO) of the South African Astronomical Observatory as part of an ongoing observing campaign to monitor the source. Complementary observations from the Steward Observatory blazar monitoring program were also described. The optical polarization variability was compared to simultaneous gamma-ray measurements as recorded by the FERMI satellite, enabling us to probe both the low- and high-energy peaks of the source's spectral energy distribution.

\section{Time-Series Data Sets at the NASA Exoplanet Science Institute}

P. Plavchan ${ }^{1}$ et al.

${ }^{1}$ NASA Exoplanet Science Institute, CalTech, Pasadena, CA 91125, USA

email: plavchan@ipac.caltech.edu

The NASA Exoplanet Science Institute (NExScI) is home to an exoplanet archive with the aim of providing support for NASA's planet finding and characterization goals. NExSCI serves as the official US portal for the public CoRoT data products. In addition, NExScI serves KEPLER public data time-series and KEPLER Object of Interest (KOI) tables, as well as synoptic data from numerous ground-based surveys. NExScI developed a periodogram service to determine periods of variability phenomena and create phased 
photometric light curves. Through the NExScI periodogram interface, the user may choose three different period detection algorithms to use on any time-series product, or even upload and analyze their own data. For more details, see http://nexsci . caltech.edu.

\title{
Search for Gravitational Waves Associated with the S5/VSR1 IPN Short Gamma-Ray Bursts
}

\author{
Valeriu Predoi ${ }^{1}$ (for LIGO Scientific Collaboration and Virgo Collaboration) \\ ${ }^{1}$ School of Physics and Astronomy, Cardiff University, Cardiff, CF24 3AA, UK \\ email: Valeriu.Predoi@astro.cf.ac.uk
}

One of the preferred progenitor models for short hard gamma-ray bursts (SHB) is the merger of compact binary objects, i.e., either neutron star-neutron star or neutron starblack hole binary systems. Such mergers are also predicted to emit strong gravitational radiation with waveforms that can be described theoretically. The poster described a new search for these known gravitational-wave signatures in temporal and directional coincidence with SHBs detected by the Interplanetary Network (IPN) satellites during LIGO's fifth science run, S5, and Virgo's first science run, VSR1. The IPN uses triangulation of relative times of arrival, so the detected bursts are localized to extended patches of the sky that vary in size and shape. The search is done fully coherently, and a new development needs to be implemented for a search on various error boxes. The poster presented the search targets, the method, and new tools that will be used to perform the search.

\section{The Low-Latency Search for Gravitational Waves from Compact Binary Coalescence}

Larry Price $^{1}$ (on behalf of the LIGO Scientific Collaboration and Virgo Collaboration)

${ }^{1}$ LIGO Laboratory, California Institute of Technology, Pasadena, CA 91125, USA

email: price_l@ligo.caltech.edu

During the summer of 2010, the first low-latency search for gravitational waves from compact binary coalescences was performed using the LIGO and Virgo instruments. The aim was to provide triggers for follow-up observations with telescopes operating in all parts of the electromagnetic spectrum. The poster described the low-latency pipeline used to produce the triggers, and the latest results from the pipeline. 


\section{The RApid Temporal Survey}

Gavin Ramsay and Tom Barclay

Armagh Observatory, College Hill, Armagh BT61 9DG

email: gar@arm.ac.uk

The Rapid Temporal Survey (RATS) explores the faint, variable sky and is sensitive to variability on time-scales ranging from a few minutes to several hours, for sources as faint as $g \sim 24$. Our survey took place between 2003 and 2010 and covered nearly 40 square degrees, with a bias towards the Galactic plane. In the first five years of observations we obtained light curves for 3 million stars and we identified over 100,000 variable stars. Although our prime goal was to detect ultra-compact binary systems, we have discovered pulsating sdB stars, white dwarfs, SX Phe and $\delta$ Scuti stars, accreting systems, flare stars and contact binaries. The poster outlined our strategy and main results to date.

\section{Machine-Learning Methods for VAST Transient Detection}

Colorado Reed ${ }^{1,2}$ et al.

${ }^{1}$ Department of Physics, University of Iowa, Iowa City, IA USA

${ }^{2}$ Jet Propulsion Laboratory, CalTech, Pasadena, CA 91109, USA.

email: colorado-reed@uiowa.edu

The Variables and Slow Transients (VAST) survey is a future radio survey for the Australian Square Kilometer Array Pathfinder (ASKAP). This survey will examine relatively long-lived (slow) astronomical transients - those lasting longer than 5 seconds. In the poster we examined machine-learning methods for the detection of slow transient events in archived light-curve data. Event detection in those light curves is inherently challenging because of uneven sampling, data sparsity, and noise from a multitude of sources. Furthermore, event duration may range from days (supernovæ) to years (novæ and intra-day variables). The poster explored supervised and unsupervised methods for event detection, and evaluated performance both on simulated VAST data and on real data from the VLA.

\section{The Delay-Doppler Spectrum of Interstellar Scintillation from Pulsars}

Barney Rickett ${ }^{1}$, Bill Coles ${ }^{1}$, Dan Stinebring ${ }^{2}$, and JJ Gao ${ }^{1}$

${ }^{1}$ University of California, San Diego, La Jolla, CA 92093, USA

email: bjrickett@ucsd.edu

2 Physics Department, Oberlin College, Oberlin, OH 44074, USA

The dynamic radio spectrum of the interstellar scintillation of radio pulsars reveals unexpected features of the turbulent ionized interstellar medium, when analyzed in the Fourier domain versus relative delay and Doppler shift. In that domain a primary "parabolic arc" is often seen. For some objects there are also reversed offset parabolæ which imply highly localized concentrations of elongated turbulent plasma. The poster presented the results and methods of analysis from two such pulsars. 


\section{How to Simulate a 10-Year LSST Program, and How to Make Sense out of What it Produces}

Stephen Ridgway, Srinivasan Chandrasekharan and the LSST Simulator Team

National Optical Astronomical Observatory, Tucson, AX 85719, USA

email: ridgway@noao.edu

A high-throughput survey for transient sources will have to deal with a significant number of transient or variable sources of familiar and less interesting types which may be difficult to distinguish from higher priority and novel sources. The two major astrophysical sources of contaminants are small-solar system bodies and Galactic variable stars. The frequency of solar-system body appearances can be estimated reasonably from existing model populations. Recently, tools and data have become available which can support an estimate of the number of stellar variable sources that will be detected at any survey depth. The essential tool for describing the stellar population is a Galactic model of stellar sources (e.g., the Besançon model). The essential data are empirical statistics on stellar variability from the KePLeR survey and the Sloan Digital Sky Survey. They were applied to the Large Synoptic Survey Telescope (LSST), for which the estimates of the number of variable target detections has ranged over at least an order of magnitude. We estimate that the LSST survey will detect variation in $\sim 10^{5}$ stars each night, and that this number is only a weak function of the accuracy of photometric calibration.

\section{Classification of Hipparcos Unsolved and Non-Investigated Variables}

L. Rimoldini ${ }^{1}$ et al.

${ }^{1}$ Observatoire astronomique de l'Université de Genève, 1290 Versoix, Switzerland email: Lorenzo.Rimoldini@unige.ch

The Hipparcos catalogue contains thousands of unclassified variable stars whose periodicity could not be determined or confirmed from the literature; to date they remain unsolved, or not investigated. The poster described our development of an automated supervised classification technique, based on Random Forests, that we trained on sources with either periodic or non-periodic light variations, in order to predict variability classes for Hipparcos variables with unreliable or missing classification.

\section{Time Domain Astronomy with JANUS}

P. Roming

Southwest Research Institute, San Antonio, TX 78228, USA

email: roming@swri.edu

The Joint Astrophysics Nascent Universe Satellite (JANUS) is a multi-wavelength cosmology mission focused on the cosmic dawn. The primary objectives of JANUS are to discover and observe high- $z(z>5)$ gamma-ray bursts (GRBs) and quasars. An inevitable by-product of searching for these high- $z$ objects is that JANUS will produce X-ray and near-IR surveys that will reveal many other types of time-domain events. Such events include detections of GRB-supernova events, low- $z$ GRBs, stellar super-flares, neutronstar super-bursts, fast X-ray transients, and "tidal disruption events" triggered by the close encounter of a star with a supermassive black hole. 


\title{
Binary Tidal Disruption by a Supermassive Black Hole: Observational Consequences
}

\author{
Elena M. Rossi \\ Leiden Observatory, NL-2333 CA Leiden, The Netherlands \\ email: emr@strw.leidenuniv.nl
}

Observations of hyper-velocity stars in the Galactic Halo and stellar orbits at the Galactic Centre may be the consequence of tidal disruption of binaries by the supermassive black hole. In this process, one star may be ejected at high velocity, while the companion remains bound to the hole. The poster first presented a new method to calculate the disruption probability of binaries which enter the tidal sphere, and their energy after disruption. It then showed our predictions for the velocity distribution of the ejected stars and for the mass distribution of the stars that remain bound. A tentative comparison with current sparse data was also made.

\section{Viscous Evolution of WD-WD Merger Remnants}

Josiah Schwab, Ken Shen, and Eliot Quataert

University of California, Berkeley, CA 94720, USA

email: jwschwab@berkeley.edu

Beginning with the results of 3D smoothed-particle hydrodynamics ( $\mathrm{SPH}$ ) simulations of merging white dwarfs, we performed $2 \mathrm{D}$ calculations of the evolution of the post-merger remnant. The poster described the operation of this project.

\section{Newly-Discovered Properties of Cepheids in the Mid-Infrared}

Victoria Scowcroft ${ }^{1}$ et al.

${ }^{1}$ Carnegie Observatories, Pasadena, CA, 91101, USA

email: vs@obs.carnegiescience.edu

The Carnegie Hubble Program (CHP) is a Warm Spitzer programme designed to measure the Hubble constant to an accuracy of $2 \%$ using Cepheids, supernovæ and the mid-infrared Tully-Fisher relation; Freedman et al. (2011; arXiv:1109.3802) present an overview of the CHP. As part of that project we have taken observations of Cepheids in the Milky Way and the Large and Small Magellanic Clouds in a deterministic manner. The stars were targeted individually; each one was observed at 24 (MW and LMC) or 12 (SMC) equally-spaced epochs through a single cycle. We were able to confirm the predicted temperature-dependent effect that the $\mathrm{CO}$ bandhead at $4.6 \mu \mathrm{m}$ has on the [4.5] magnitude (Marengo et al. 2010, ApJ 709, 120), both through the Cepheid's pulsation cycle and in the period-luminosity (PL) relation.

We find that the 3.6- $\mu \mathrm{m}$ PL relation is a good distance indicator; reddening effects are drastically reduced compared to the optical region, and metallicity differences do not appear to have any influence. In the $4.5-\mu \mathrm{m}$ PL relation, and additionally in the [3.6]-[4.5] period-colour relation, metallicity does appears to play a role. The amplitude of the effect of the CO bandhead on [4.5] is different between the three galaxies, altering the mean 
colour of the Cepheids and affecting the amplitude of the Cepheid light curves. We are in the process of using these data to calibrate the photometric metallicity-colour relation and of applying the technique to Cepheids that are not accessible spectroscopically.

The work presented in this poster is being published by Scowcroft et al. (arXiv:1108:4672).

\section{Numerical Simulations of Line-Profile Variation Beyond a Single- Surface Approximation for Oscillations in RoAp Stars}

Hiromoto Shibahashi amd Jun Naito

Department of Astronomy, University of Tokyo, Tokyo 113 0033, Japan

email: shibahashi@astron.s.u-tokyo.ac.jp

Prior to the last decade, most observations of roAp stars concerned their light variations. Recently some new, striking results of spectroscopic observations with high timeresolution, high spectral dispersion and high signal-to-noise ratios became available. Since the oscillations found in roAp stars are high overtones, the vertical wavelengths of the oscillations are so short that the amplitude and phase of variation of each spectroscopic line are highly dependent on the level of the line profile. Hence the analyses of variation of spectroscopic lines of roAp stars potentially provide us with new information about the vertical structure of the atmosphere of these stars. In order to extract such information, numerical simulation of line-profile variations beyond a single-surface approximation is necessary. We have carried out numerical simulations of line-profile variations by taking into account the finite thickness of the line-forming layer. The poster demonstrated how effective that treatment is, by comparing the simulations with the observed line profiles.

\section{Detecting Variability in Astronomical Time-Series Data: Applications of Clustering Methods in Cloud Computing Environments}

Min-Su Shin

Department of Astronomy, University of Michigan, Ann Arbor, MI 48109, USA email: msshin@umich.edu

The poster presented applications of clustering and de-trending methods to detect variability in massive astronomical time-series data. Focusing on variability of bright stars, we used clustering methods to separate possible variables from other time-series sources such as intrinsically non-variable objects and sources with common systematic patterns. We tested how de-trending of systematic patterns can be incorporated into our variability detection approach. The study was conducted in a cloud computing environment provided by KISTI. The poster described our experience using the cloud computing test-bed. 


\section{The Collaboration for Astronomy Signal Processing and Electronics Research}

Andrew Siemion, Dan Werthimer and The CASPER Collaboration

University of California, Berkeley, Berkeley, CA 94720, USA

email: siemion@berkeley.edu

The poster presented the latest updates on the open source digital signal processing hardware and software being developed by the Collaboration for Astronomy Signal Processing and Electronics Research (CASPER). The CASPER toolkit now includes Xilinx Virtex6 field programmable gate array (FPGA)-equipped Reconfigurable Open Architecture Computing Boards (ROACH II), as well as infrastructure for heterogenous architectures made up of FPGAs, graphics processing units (GPUs) and commodity CPUs. These tools enable rapid design of high-performance instrumentation by non-experts, enabling the performance of specialized instruments to track closely Moore's law for growth in the electronics industry.

\section{Technique for Low-Latency Detection of Compact Binary Coalescence and its Implications for Multi-Messenger Astronomy}

Leo Singer and N. Fotopoulos

California Institute of Technology, Pasadena, CA 01125, USA

email: singer_l@ligo.caltech.edu

The rapid detection of compact binary coalescence with a network of advanced gravitational-wave detectors will offer a unique opportunity for multi-messenger astronomy. Prompt detection alerts to the astronomical community may make it possible to observe the onset of electromagnetic emission from compact binary coalescence. The poster demonstrated a computationally practical analysis strategy which could be used to produce early-warning triggers for astronomical observatories. The work it described was carried out in collaboration with Kipp Cannon, Romain Cariou, Adrian Chapman, Mireia Crispin-Ortuzar, Melissa Frei, Chad Hanna, Erin Kara, Drew Keppel, Laura Liao, Stephen Privitera, Antony Seale and Alan Weinstein. A paper describing the results in full is now being published (arXiv1107.2665).

\section{Hitting the JACPOT: Current and Future Probes of the Accretion Disk-Radio Jet Coupling of X-Ray Binaries}

G.R. Sivakoff ${ }^{1}$ and the JACPOT XRB Collaboration

${ }^{1}$ University of Alberta, Edmonton, Alberta, T6G 2G\%, Canada

email: sivakoff@ualberta.ca

The accretion disks and radio jets of X-ray binaries are known to be coupled; however, until recently the prevailing paradigm had not been tested with direct high-resolution imaging of the radio jet over entire outbursts. Moreover, such observations had not previously targeted X-ray binaries where the compact object was either a neutron star or a white dwarf. The Jet Acceleration and Collimation Probe Of Transient X-Ray Binaries (JACPOT XRB) team has recently concluded its first monitoring series, including VLA, VLBA, X-ray, optical and near-IR observations, of entire outbursts of X-ray binaries 
with a black hole candidate (H1743-322), a neutron star (Aquila X-1) and a white dwarf (SS Cyg). The poster concentrated on the results for H1743-322 and Aql X-1. While we largely confirmed the accretion-disk radio jet paradigm, we also discovered new intriguing behaviours. Neutron stars appear as capable as are black holes at launching jets, but the lack of ejecta in Aql X-1 may suggest a fundamental difference in the jet formation process between neutron star and black hole systems, possibly hinting at an additional role from the ergosphere. The poster discussed how future radio observatories would extend our capacity to study the accretion-disk radio-jet connection.

\section{The Liverpool Telescope}

\section{A. Steele}

Astrophysics Research Institute, LJMU, Birkenhead, CH41 1LD, UK

email: ias@astro.livjm.ac.uk

The Liverpool Telescope is a 2.0-metre fully-robotic telescope sited on La Palma. It specialises in time-domain astrophysics, with strong programmes in SNe, GRBs, exoplanets and QSO monitoring. The telescope is equipped with an optical imaging camera, an integral-field fibre-fed optical spectrograph, a fast-readout wide field camera, a lucky imaging camera and an imaging polarimeter. All instruments are mounted on the telescope at all times, and can be switched over in under 20 seconds. Future instrument plans include a dual beam optical/IR tip-tilt corrected imaging camera, and a multiband imaging polarimeter.

\section{DASCH variables in the KEPLER field}

Sumin Tang ${ }^{1}$, Jonathan Grindlay ${ }^{2}$, Edward $\operatorname{Los}^{2}$, and Mathieu Servillat ${ }^{2}$

${ }^{1}$ Harvard University, Cambridge, MA 02138, USA

email: stang@cfa.harvard.edu

${ }^{2}$ Harvard-Smithsonian Center for Astrophysics, Cambridge, MA 02138, USA

DASCH is a project to digitize and analyze the scientific data contained in the $\sim 550,000$ Harvard College Observatory plates taken between the 1880s and 1990s. The collection is a unique resource for studying temporal variations in the universe over time-scales of 10-100 years. The poster presented a few of the most interesting long-term variables which we found in or near the KEPLER Field, including a group of Be variables showing variations of about 1 mag over years, a group of $\mathrm{K}$ giants showing slow variations over 10-100 years, and two other examples. 


\section{Towards Higher Precision on Time-Series Differential Photometry through Colour Effects Minimization}

Victor Terron ${ }^{1}$, Matilde Fernandez ${ }^{1}$, and Monika G. Petr-Gotzens ${ }^{2}$

${ }^{1}$ Institute of Astrophysics of Andalusia, IAA-CSIC, 18008 Granada, Spain

email: vterron@iaa.es

${ }^{2}$ European Southern Observatory, D-85748 Garching, Germany

Common knowledge of differential photometry establishes that in order to improve the photometric precision of light curves it is essential to follow several rules. First and foremost, stars must be placed in the same pixels of the CCD and their light distributed over several of them, while the aperture size must be optimized. Furthermore, algorithms based on a careful selection of the comparison stars, depending on their noise level, go one step further towards achieving the maximum accuracy. Nevertheless, astronomers must still address the fact that, particularly when observing at high air-masses, atmospheric extinction does not affect all the stars equally; blue stars appear dimmer than red ones. Therefore, as the stars in the field of view drift across the sky, going through different air-masses, they suffer from different extinction. As a result a comparison star may look relatively brighter or fainter than it really is. The algorithm that we proposed in the poster tackles this matter by selecting an artificial comparison star for each target so that the colour effects of atmospheric extinction can be minimized. In that manner, and especially in the case of well populated fields, the effects of atmospheric extinction are considerably reduced. Although some issues remain open, we believe that our algorithm would be of decisive help for high-precision photometry with ground-based telescopes.

\section{High-redshift Type II Supernova Survey with Shock Break-out}

Nozomu Tominaga ${ }^{1,2}$

${ }^{1}$ IPMU, University of Tokyo, Chiba 27r-8583, Japan

${ }^{2}$ Department of Physics, Konan University, Kobe, Hyogo 658-8501, Japan

email: tominaga@konan-u.ac.jp

Shock break-out is the brightest radiative phenomenon in a supernova (SN), but is difficult to observe owing to its short duration and X-ray/UV-peaked spectra. First observations of shock break-out were reported in 2008 for SN 2008D (Type Ib) in X-ray and optical data from SwIFT, and in SNLS-04D2dc and SNLS-06D1jd (both (Type II $\mathrm{SNe}$ ) in UV-optical observations made by GALEX and the Supernova Legacy Survey (SNLS). We have constructed a SN II model with a multigroup radiation hydrodynamics code STELLA, which reproduces well the UV-optical multicolour light curves of SNLS04D2dc. It demonstrates that the peak apparent $g$-band magnitude of the shock breakout would be $m_{g}=26.4$ if an SN identical to SNLS-04D2dc occurred at a red-shift of $z=1$ (which can be reached by 8 -m-class telescopes). We also constructed shock breakout models for SNe II with various main-sequence masses, metallicities and explosion energies, and estimated the observable SN rate and attainable red-shift by convolving an initial mass function with the cosmic star-formation history, intergalactic absorption and host-galaxy extinction; the observable SN rate in the $g$-band for $m_{g^{\prime}, \text { lim }}=27.5 \mathrm{mag}$ is $3.3 \mathrm{SNe} \mathrm{deg}^{-2} \mathrm{day}^{-1}$. Half will be found at $z \geqslant 1.2$. That estimate enabled us to propose a realistic survey strategy optimized for shock break-out; multicolour observations in the blue optical bands with $\sim 1$ hour intervals are essential for the efficient detection, identification and interpretation of the shock break-out. The poster emphasized that shock break-out is an advantageous tool for probing high- $z$ Type II SNe. 
Tidal Resonance in Short Gamma-Ray Burst Precursors and Multimessenger Constraints on Neutron Star Physics

David Tsang ${ }^{1}$, Jocelyn Read ${ }^{2}$, Tanja Hinderer ${ }^{1}$, and Tony Piro ${ }^{3}$

${ }^{1}$ California Institute of Technology, Pasadena, CA 91125, USA

email: dtsang@caltech.edu

${ }^{2}$ University of Mississippi, Oxford, MS 38677, USA

${ }^{3}$ University of California, Berkeley, CA 94720, USA

The merger of a neutron star with another compact object is a leading model for the progenitor of a short Gamma-Ray Burst (sGRB). The gravitational waves which drive their inspiral are expected to be observed by ground-based gravitational-wave detectors such as the upcoming Advanced LIGO. The poster examined the last seconds of coalescing binary neutron stars to understand possible sources of X-ray and gamma-ray emission. It described how precursor observations can potentially constrain the structure and equation of state of component neutron stars. Tidal effects are the strongest source of perturbation in this régime. However, the strength of the tidal field is insufficient to break the crust directly until milliseconds before the merger. Resonant modes that are concentrated near the crust-core interface can be excited to large amplitudes, fracturing the crust and leading to a pre-merger signature seconds before the merger itself. The timing of such a precursor flare would measure the resonant frequency of the interfacial mode, probing the structure and equation of state near the base of the neutron-star crust. This is complementary to studying the GW inspiral signal alone; the latter is primarily sensitive to the equation of state in the core, which determines NS mass and radius.

\section{Understanding Pulsation in Luminous M Supergiants with the Aid of Archival Observations}

D. G. Turner ${ }^{1}$ et al.

${ }^{1}$ Saint Mary's University, Halifax, Nova Scotia, B3H 3C3, Canada

email: turner@ap.smu.ca

The poster presented a summary of ongoing and archival photometric and radial-velocity observations of the $9^{\text {th }}$-magnitude F9 Ib supergiant HDE 344787 that were made over the last 120 years, with emphasis on recent trends. HDE 344787 is a double-mode Cepheid variable with a period of 5.4/3.8 days; it has extremely small amplitude $(<0.01$ magnitude) with a rapidly-increasing period and sinusoidal light variations of decreasing amplitude that may result in non-variable status within the next half century. The star displays all of the characteristics of a Cepheid that is undergoing a first crossing of the instability strip and is about to depart the cool edge for first crossers. Its existence helps to redefine the observational characteristics normally attributed to Cepheid pulsation. 


\section{Detecting Stellar Tidal Disruptions: from Optical to Radio}

S. van Velzen, E. Koerding, and H. Falcke

IMAPP, Radboud University, 6500 GL Nijmegen, the Netherlands

email: s.vanvelzen@astro.ru.nl

When a star passes too close to a massive black hole it becomes tidally disrupted. About 10 examples of the flares that occur when the debris of the disruption falls back onto the black hole have been identified in UV and X-ray surveys. The discovery of two tidal-flare candidates in archived SDSS data implies that we can expect tens to hundreds of such events per year from current and near-future optical variability surveys. To explore the potential of detecting tidal disruptions with radio surveys, we used the well-established scaling between accretion power and jet luminosity to construct a robust model of the emission of a tidal disruption jet. The poster illustrated how we reproduced the radio flux of the recent tidal-flare candidate GRB 110328A, and described our finding that future radio surveys will be able to test whether the majority of tidal disruptions are accompanied by a relativistic jet.

\section{IAU "SOFA": Time-scale Transformation Software}

Patrick Wallace

STFC Rutherford Appleton Laboratory, Didcot, OX11 0QX, UK

email: Patrick.Wallace@stfc.ac.uk

The software provided through the IAU "Standards of Fundamental Astronomy" (SOFA) initiative now includes a suite of time-scale transformation functions, supporting TAI, UTC, UT1, TT, TCG, TCB and TDB. The SOFA design features two-part Julian dates (to safeguard precision), and individual treatment of specific transformations rather than a single general-purpose routine. Correct handling of UTC leap seconds was the biggest single challenge.

\section{AGILITE: An ATA Survey to Characterize the Population of Galactic Radio Transients and Variables}

Peter K. G. Williams and Geoffrey C. Bower

Department of Astronomy, University of California, Berkeley, CA 94720, USA

email: pwilliams@astro.berkeley.edu

Systematic studies of transient and variable radio emission are a relative novelty. Searches for "slow" radio transients - sources that vary over time-scales of days to months - have so far tended to focus on extragalactic fields. Many Galactic sources, however, vary on those time-scales, including X-ray binaries, brown dwarfs, and several objects of unknown nature discovered in previous searches. The poster presented AGILITE, the ATA Galactic Lightcurve and Transient Experiment, an effort to characterize the population of Galactic radio transients and variables more fully. AGILITE has a large overall footprint $(\sim 25$ $\left.\operatorname{deg}^{2}\right)$, a substantial number of epochs $(\sim 200)$, and a significant dedication of observatory time $(\sim 1700$ hours over two years), all of which make it sensitive to rare objects as well as to variability on many time-scales. The poster described the AGILITE pipeline and presented preliminary results from 28 observations of a field centred on the Cygnus X-3 high-mass X-ray binary, including epochs during the major flare of 2011 March. It also 
discussed prospects for the complete survey as well as other applications of the dataset, such as large-scale mapping of extended radio emission toward the Galactic Center.

\section{ThunderKAT: Radio Transients with MeerKAT}

Patrick Woudt ${ }^{1}$, Rob Fender ${ }^{2}$ and the ThunderKAT team

${ }^{1}$ University of Cape Town, Rondebosch 7701, South Africa

email: pwoudt@ast.uct.ac.za

${ }^{2}$ School of Physics \& Astronomy, University of Southampton, SO17 1BJ, UK

In 2010 MeerKAT (South Africa's SKA pathfinder telescope) selected ten Large Survey Projects, which collectively were allocated $70 \%$ of the observing time in the first 5 years of full operation of MeerKAT. ThunderKAT is the MeerKAT Large Survey Project which studies all aspects of transient radio (synchrotron) emission associated with accretion and explosive events, including relativistic jets, nova explosions, supernovæ and gamma-ray bursts. ThunderKAT's mode of observing will be two-fold; it will be fully commensal with all other Large Survey Projects on MeerKAT, interrogating the data stream at 1-second intervals with the goal of real-time transient detections. In addition a nominal 3000 hours have been reserved in the first five years of operation of MeerKAT for ThunderKAT to study the observed radio transients in detail. Science commissioning observations of transients using the KAT-7 array were planned for the second half of 2011, as part of the pathway to real-time transient detections on MeerKAT.

\section{Exploring Variability of Bright Stars on the Ecliptic Plane with STEREO}

K. T. Wraight ${ }^{1}$, G. J. White ${ }^{2}$, D. Bewsher ${ }^{3}$, and A. J. Norton ${ }^{1}$

${ }^{1}$ Dept. of Physical Sciences, The Open University, Milton Keynes, MK' 6AA, UK

email: k.t.wraight@open.ac.uk

${ }^{2}$ STFC Rutherford Appleton Laboratory, Didcot, OX11 0QX, UK

${ }^{3}$ Jeremiah Horrocks Institute, University of Central Lancashire, Preston, PR1 2HE, UK

The STEREO mission's Heliospheric Imagers have considerable potential for use in a wide range of stellar variability studies. With photometry of $\sim 900,000$ stars from $12^{\text {th }}$ to $2^{\text {nd }}$ magnitude over a five-year period within $10^{\circ}$ of the Ecliptic Plane, the HI- 1 imagers have the capability of finding many new and interesting variables, and of improving considerably the quality of available data for previously known variables in this poorlystudied part of the sky. The moderate cadence of $40 \mathrm{~min}$, maintained for $\sim 20$ days at a time and with repeated visits from each satellite, permits a wide range of potential applications across very different time-scales. Initial analyses of HI-1A data have revealed 122 new eclipsing binaries. Ongoing work includes the determination of rotation rates of magnetic CP stars and the extraction of pulsational modes from bright $\delta$ Scuti variables. The initial analyses were published in 2011 by Wraight, K.T., White, Glenn J., Bewsher, D., \& Norton, A.J. (MNRAS, 416, 2477). 


\section{Pair-Instability Supernovæ:A Second Candidate from the PTF Survey}

Ofer Yaron, Avishay Gal-Yam, et al.

Weizmann Institute of Science, Rehovot 76100, Israel

email: ofer.yaron@weizmann.ac.il

Massive metal-poor stars, attaining core masses in excess of $\sim 50 M_{\odot}$ sun, may explode via the pair-production instability. The massive oxygen-rich cores of such stars reach high temperatures $\left(>10^{9} \mathrm{~K}\right)$ at relatively low densities, such that pressure-supporting photons are converted to electron-positron pairs. A violent dynamical collapse ensues, triggering a powerful nuclear explosion, which leads to a complete disruption of the star. This notion has been theoretically predicted and understood for almost four decades, but only recently did we begin to obtain more convincing observational proof for the existence of such events. The poster presented additional data and analysis for the single most promising pair-instability supernova (PISN) candidate to date ( SN2007bi), and reported the discovery of a second candidate (PTF10nmn), first detected in 2010 May by the Palomar Transient Factory survey. The observed characteristics of PTF10nmn closely resemble those of SN 2007bi in both light curve and spectroscopic signatures. The poster showed the spectral evolution of PTF10nmn, from around peak light till the beginning of a nebular phase. A nebular model for our last spectrum of 2011 July 3, corresponding to an epoch of $>450$ days from explosion, revealed a mass of the order of $5 M_{\odot}$ of ejected ${ }^{56} \mathrm{Ni}$, high ejected masses of $\mathrm{C}, \mathrm{O}, \mathrm{Ca}$ and $\mathrm{Si}$, and a total ejecta mass $M_{e j}$ of $\sim 100 M_{\odot}$. The seemingly real double peak (with a rest-frame separation of $\sim 50$ days) as apparent from the $R$-band photometry, is in itself an intriguing finding that requires a suitable physical explanation.

\section{"WISEASS" — A State-of-the-Art Interactive Spectroscopic Database}

Ofer Yaron, Avishay Gal-Yam, et al.

Weizmann Institute of Science, Rehovot 76100, Israel

email: ofer.yaron@weizmann.ac.il

The poster reported the development of a new spectroscopic database, the Weizmann Institute of Science Experimental Astrophysics Spectroscopy System (WISEASS). The system consists of a MYSQL DB and a web-interface that is implemented mainly in PHP, embedded within a DRUPAL (CMS) framework. The purpose of this system is to serve as an archive of high-quality supernovæ spectra, with respect to both historical ("legacy") data, and data that are accumulated in ongoing modern surveys and programmes (both currently and in the future). Besides serving solely as an archive, with the ability to hold and retrieve spectra and objects alongside all related information (via numerous querying options), we have implemented means by which it is possible to carry out basic online analyses of the spectra. By making use of interactive plots, with zooming and over-plotting capabilities, the database provides graphical interfaces for performing line identifications of the major relevant species, assessing red-shifts and expansion velocities. The Database currently includes $\sim 3000$ spectra, of which $\sim 2000$ are public, the latter including, for example, published PTF spectra and all of the "SUSPECT" archive. Owing to the increasing interest and use of this system by the SN communities world-wide, it is expected that additional programme and data from various sources and projects will be added and incorporated in the near future. 


\section{The Unusual Gamma-Ray Transient Swift J164449.3+573451}

B. Ashley Zauderer

Harvard-Smithsonian Center for Astrophysics, Cambridge, MA 02138, USA email: bzauderer@cfa.harvard.edu

The poster presented data from an observational campaign at radio wavelengths following discovery of the unusual transient source, Swift J164449.3+573451 on 2011 March 25. What made this source unusual was the magnitude and duration of X-ray flaring after the initial burst. The observations described spanned centimetre to sub-mm wavelengths, establishing spatial coincidence with the nucleus of an inactive galaxy at $z=0.354$. The radio light curves and spectral energy distributions are consistent with the theoretical model of accretion from a tidal disruption event onto a $10^{6}-M_{\odot}$ black hole launching a new relativistic jet. Monitoring this event at radio wavelengths after the initial concerted campaign has continued, and the poster concluded with a summary of the observations to date. 